\title{
Research Article \\ Effects of Aluminum on Hydrogen Solubility and Diffusion in Deformed Fe-Mn Alloys
}

\author{
C. Hüter, ${ }^{1,2}$ S. Dang, ${ }^{1}$ X. Zhang, ${ }^{2}$ A. Glensk, ${ }^{2}$ and R. Spatschek ${ }^{1,2}$ \\ ${ }^{1}$ Institute for Energy and Climate Research, Forschungszentrum Jülich GmbH, 52425 Jülich, Germany \\ ${ }^{2}$ Computational Materials Design Department, Max-Planck Institut für Eisenforschung, 40237 Düsseldorf, Germany \\ Correspondence should be addressed to R. Spatschek; r.spatschek@fz-juelich.de
}

Received 15 May 2016; Accepted 7 August 2016

Academic Editor: Pavel Lejcek

Copyright (c) 2016 C. Hüter et al. This is an open access article distributed under the Creative Commons Attribution License, which permits unrestricted use, distribution, and reproduction in any medium, provided the original work is properly cited.

\begin{abstract}
We discuss hydrogen diffusion and solubility in aluminum alloyed Fe-Mn alloys. The systems of interest are subjected to tetragonal and isotropic deformations. Based on ab initio modelling, we calculate solution energies and then employ Oriani's theory which reflects the influence of $\mathrm{Al}$ alloying via trap site diffusion. This local equilibrium model is complemented by qualitative considerations of Einstein diffusion. Therefore, we apply the climbing image nudged elastic band method to compute the minimum energy paths and energy barriers for hydrogen diffusion. Both for diffusivity and solubility of hydrogen, we find that the influence of the substitutional $\mathrm{Al}$ atom has both local chemical and nonlocal volumetric contributions.
\end{abstract}

\section{Introduction}

High strength structural steels are a material class key to further developments in automotive light-weight construction [1-3]. At Mn weight fractions between 15 and 25 percent, high manganese steels offer a combination of high strength and desirable plasticity characteristics. The stable austenitic structure offers high cost efficiency [4-8], and investigations on Fe-Mn systems revealed the relation of high work hardening rates and ductility to the austenitic microstructure $[9,10]$. In addition, high manganese steels exhibit a lower susceptibility to hydrogen embrittlement [11] than ferritic and martensitic steels $[12,13]$.

The systematic investigation of hydrogen embrittlement in high manganese steels is challenged by the microstructural, interfacial, and chemical complexity of these materials. The scientific focus has been on intergranular failure modes and general heterophase interface failure [14-18]. Hydrogen induced degradation [19] is a major hindrance for the implementation of promising steel designs for automotive applications [20]. While the austenitic phase itself exhibits low diffusivity and high solubility of hydrogen, the transformation induced plasticity (TRIP) moderated martensitic transformation yields strong gradients of solubility and diffusivity due to the presence of ferritic phases.
An improved resistance to hydrogen induced delayed fracture can be realised by adding temper softeners like $\mathrm{Cr}$, $\mathrm{Mo}$, and $\mathrm{V}$ in martensitic steels. This highly desirable effect originates from the suppressed fracture by carbide precipitation, shifting the intergranular fracture to intragranular fracture [21]. In austenitic steels, the addition of $\mathrm{Ni}$ and $\mathrm{Si}$ is an established method to increase the ductility of hydrogenloaded steels [22-25], and the beneficial effect of $\mathrm{Mn}$ on hydrogen solubility has been investigated for instance in [26, 27]. The successful alloying of high manganese steels with $\mathrm{Al}$, which reduces the weakness against hydrogen embrittlement, suggests studying the effect of $\mathrm{Al}$ on the different aspects of hydrogen in Fe-Mn systems. As it is an integral part of production processes, we also study the influence of mechanical loading, in practice isotropic and tetragonal distortions, on solubility and mobility of hydrogen.

The article is organized as follows. In Section 2 we describe the methodical basis of our approach, including several aspects of the systematic approximations we make. Section 3 contains the results of our investigations, where we distinguish between stress-free, isotropically distorted, and tetragonally distorted systems and discuss their influence on hydrogen solubility and diffusivity. Finally we conclude with a brief discussion of the presented results in the context of different material configurations and future perspectives. 


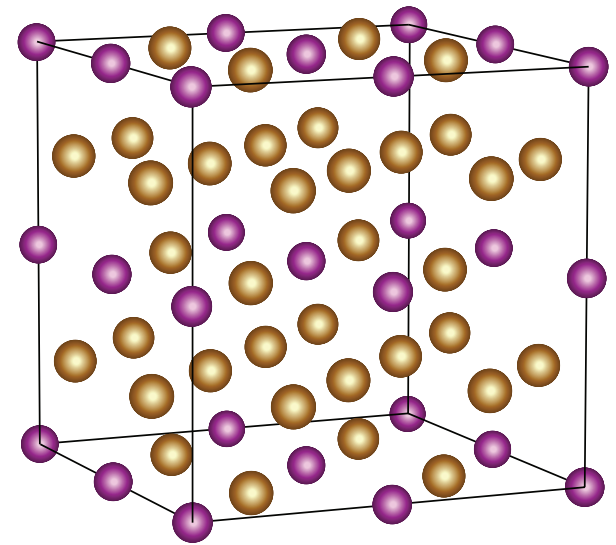

Figure 1: The supercell of the austenitic Fe-Mn system; Fe atoms are shown in yellow and $\mathrm{Mn}$ atoms in purple. The distribution of the $\mathrm{Mn}$ atoms yields only two types of octahedral interstices and one inequivalent site for the substitution $\mathrm{Fe} \rightarrow \mathrm{Al}$ which makes it an ideal reference for the study of the $\mathrm{Fe}_{23} \mathrm{Mn}_{8} \mathrm{Al}$ system.

\section{Methods}

The methodical basis of our investigation is electronic structure density functional theory (DFT). It allows predicting the quantities of interest for exactly defined crystal geometries and chemical compositions. The systems which we investigate are introduced in Figures 1 and 2 . They consist of $2 \times 2 \times 2$ supercells of an austenitic $\mathrm{Fe}_{24} \mathrm{Mn}_{8}$ matrix and the resulting system when we substitute one $\mathrm{Fe}$ atom by an $\mathrm{Al}$ atom, $\mathrm{Fe}_{23} \mathrm{Mn}_{8} \mathrm{Al}$. Hydrogen is introduced on interstitial sites. We obtained our $a b$ initio results from DFT calculations employing the Vienna $a b$ initio simulation package (VASP) [28]. For the PAW pseudopotentials [29] we use the GGA-PBE exchange and correlation functional [30]. The convergence of DFT related parameters has been carefully tested. We find that an $8 \times 8 \times 8 \mathrm{k}$-point Monkhorst-Pack [31] mesh for the 2 $\times 2 \times 2$ supercell and a plane wave energy cutoff of $600 \mathrm{eV}$ are sufficient to ensure a convergence of the calculated formation energies to better than $10 \mathrm{meV}$. An electronic smearing of $0.1 \mathrm{eV}$ was chosen within the Methfessel-Paxton scheme [32]. Further, subject-specific numerical details will be discussed at the appropriate places of this publication.

\section{Results}

3.1. Hydrogen Solubility. The goal of this section is to determine the concentration of hydrogen as solid solution in the fcc Fe-Mn-Al system. Two aspects are of primary interest here: first, the local arrangement of the hydrogen atoms in the interstitial sites in different environments of iron, manganese, and aluminum, to find a site preference for the hydrogen; second, the dependence of the solubility on mechanical deformation conditions, in particular for pure volume changes due to isotropic straining and for volumeconserving tetragonal distortions.

The central quantity required to determine the equilibrium hydrogen concentrations is the solution energy, which is defined as

$$
E^{\sigma}=E_{\mathrm{MH}}[\sigma]-E_{\mathrm{M}}-\frac{1}{2} E_{\mathrm{H}_{2}}
$$

TABLE 1: The solution enthalpies of hydrogen at the four investigated octahedral sites and in the Fe-Mn reference system. The second row describes the nearest neighbour (n.n.) chemical environment of the hydrogen atom in the octahedral position, where, for example, 4-2-0 denotes that it is surrounded by $4 \mathrm{Fe}, 2 \mathrm{Mn}$, and no $\mathrm{Al}$ atoms.

\begin{tabular}{lccccc}
\hline$\sigma$ & Fe-Mn & oc1 & oc2 & oc3 & oc4 \\
\hline n.n. $(\mathrm{Fe}-\mathrm{Mn}-\mathrm{Al})$ & $4-2-0$ & $3-2-1$ & $4-2-0$ & $5-0-1$ & $6-0-0$ \\
$E^{\sigma}(P=0)(\mathrm{eV})$ & 0.054 & 0.140 & -0.010 & 0.130 & 0.073 \\
\hline
\end{tabular}

Here, $E_{\mathrm{MH}}[\sigma]$ is the energy of the metal-hydrogen system, where $\sigma$ denotes the occupied interstitial site of the hydrogen, as illustrated in Figure 2. $E_{\mathrm{M}}$ is the energy of the purely metallic alloy system, and $E_{\mathrm{H}_{2}} / 2$ is the reference potential for half an isolated $\mathrm{H}_{2}$ molecule. We note that for vanishing external stress the solution energy and enthalpy coincide. This energy difference $E^{\sigma}$ will in the following be computed using $a b$ initio techniques. It serves as an input for the calculation of the equilibrium hydrogen concentration (or occupation probability for the different interstices), which is in the dilute limit of noninteracting hydrogen atoms given by Sievert's law [33]:

$$
c_{\mathrm{H}} \sim p_{\mathrm{H}_{2}}^{1 / 2} e^{-E^{\sigma} / k_{B} T}
$$

where $p_{\mathrm{H}_{2}}$ is the partial pressure of the surrounding $\mathrm{H}_{2}$ gas. We have to distinguish this pressure from a mechanical pressure $P$ acting on the solid solution phase, which can be independently controlled by mechanical constraints. In the following, we will focus on the mechanical part and not further discuss the gas pressure $p_{\mathrm{H}_{2}}$. The value of the (suppressed) proportionality constant in the above expression (2) depends on the choice of a reference pressure and the precise definition of the hydrogen concentration.

3.1.1. Stress-Free Systems. We calculate the energy contributions $E_{\mathrm{MH}}$ and $E_{\mathrm{M}}$ for the supercells shown in Figures 1 and 2, using full ionic relaxation and vanishing external (mechanical) pressure, $P=0$. In this case the solution energies are identical to the solution enthalpies. The resulting values are given in Table 1.

For the Fe-Mn reference system we only consider those octahedral sites which exhibit the energetically most favorable chemical neighbourhood, where the hydrogen atom is surrounded by 4 iron and 2 manganese atoms; see Figure 1. Such a site exists 24 times in the $\mathrm{Fe}_{24} \mathrm{Mn}_{8}$ supercell. The other type of octahedral sites, where the $\mathrm{H}$ atom is surrounded by 6 Fe atoms, exists 8 times but has a higher solution energy when the system is deformed.

This energy is compared to different atomic arrangements for a $\mathrm{Fe}_{23} \mathrm{Mn}_{8} \mathrm{Al}$ system, where one iron atom is replaced by aluminum. For this configuration, there are four chemically inequivalent environments for the $\mathrm{H}$ atom, as depicted in Figure 2. For the configuration denoted by "ocl" the nearest neighbours of the $\mathrm{H}$ atom are $3 \mathrm{Fe}$ and $2 \mathrm{Mn}$ atoms and one $\mathrm{Al}$ atom, which exists four times in the supercell. The configuration "oc2" has the neighbourhood $4 \mathrm{Fe}, 2 \mathrm{Mn}$, and 0 $\mathrm{Al}$, with a multiplicity of 20. "oc3" has the environment $6 \mathrm{Fe}$, 

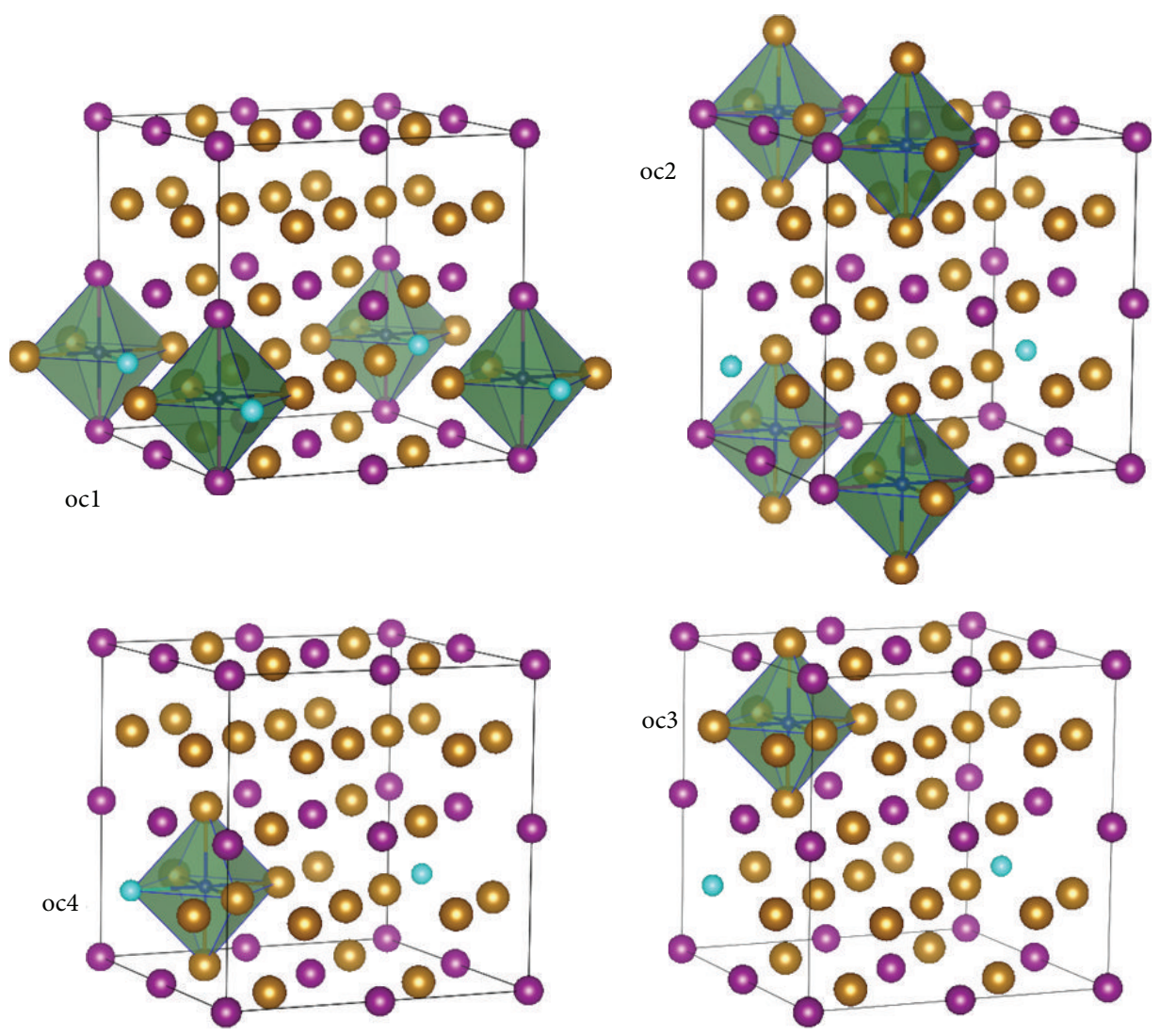

FIGURE 2: The $\mathrm{Fe}_{23} \mathrm{Mn}_{8} \mathrm{Al}$ austenitic supercell. Fe atoms are yellow, Mn atoms are purple, and blue marks Al. The four different octahedral sites we consider are highlighted by polyhedra. The hydrogen atom can be located in direct neighbourhood of $3 \mathrm{Fe}, 2 \mathrm{Mn}$, and $1 \mathrm{Al}$ atoms ("oc1"), $4 \mathrm{Fe}$ and $2 \mathrm{Mn}$ atoms ("oc2"), $6 \mathrm{Fe}$ atoms ("oc3"), or $5 \mathrm{Fe}$ and $1 \mathrm{Al}$ atom ("oc4"). Not shown here are the tetrahedral sites. We note that the oc 2 configuration has the same next neighbour configuration as our reference octahedral site in the $\mathrm{Fe}_{24} \mathrm{Mn}_{8}$ system, $4 \mathrm{Fe}$ and $2 \mathrm{Mn}$ atoms, but differs by the substitution of one Fe by Al. The ocl case corresponds to the substitution of one Fe atom at the reference site with an $\mathrm{Al}$ atom, changing the next neighbourhood configuration as $4 \mathrm{Fe} 2 \mathrm{Mn} \rightarrow 3 \mathrm{Fe} 2 \mathrm{Mn} 1 \mathrm{Al}$.

$0 \mathrm{Mn}$, and $0 \mathrm{Al}$, and it exists six times. Finally, "oc4" has $5 \mathrm{Fe}$, $0 \mathrm{Mn}$, and $1 \mathrm{Al}$ neighbours and exists twice. Apart from the chemical environment, also the local geometric arrangement influences the solution energies. In the case of the "oc2" configuration, which is of specific interest due to its energetic preferability, we find the most attractive local arrangement in 9 of the 20 sites. The resulting 11 sites are less attractive and split into three subgroups of local geometric arrangements. The resulting solution energies for the most attractive sites for each chemical environment are listed in Table 1.

By comparison to the reference system $\mathrm{Fe}_{24} \mathrm{Mn}_{8}$, we see that there are two types of interstices with identical next neighbour configuration there and in $\mathrm{Fe}_{23} \mathrm{Mn}_{8} \mathrm{Al}$. The oc2 site is energetically more favorable by about $60 \mathrm{meV}$ than the reference site with $4 \mathrm{Fe}$ and $2 \mathrm{Mn}$ atoms as nearest neighbour. This is attributed to the nonlocal effect of the substitutional $\mathrm{Al}$ atom. The oc3 site is about $70 \mathrm{meV}$ less attractive than the reference site with $6 \mathrm{Fe}$ atoms as nearest neighbour, which has nearly the same energy as the reference site with $4 \mathrm{Fe}$ and $2 \mathrm{Mn}$ atoms in the immediate neighbourhood. This discrepancy shows that the solution energy is lowest in a manganese rich environment, which is in agreement with the predictions in $[26,27]$. It also exhibits that, in the $\mathrm{Fe}_{23} \mathrm{Mn}_{8} \mathrm{Al}$ system, the volumetric effect of the Mn atoms is limited to the direct vicinity of the Mn atoms.

The general observation is, therefore, that $\mathrm{Al}$ atoms as next neighbour make octahedral sites energetically less favorable. We can therefore conclude that for low temperatures and hydrogen concentrations the sites close to aluminum atoms will not be populated. In the room temperature regime with $k_{B} T \approx 25 \mathrm{meV}$ the occupation probability ratio of the energetically most attractive oc2 configuration of the Fe-Mn$\mathrm{Al}$ system in comparison to the $\mathrm{Fe}-\mathrm{Mn}$ reference system is therefore according to (2) given by

$$
\frac{c_{\mathrm{oc} 2}}{c_{\mathrm{ref}}}=r_{\mathrm{oc} 2 / \mathrm{ref}} \exp \left(\frac{E^{\mathrm{oc} 2}-E^{\mathrm{ref}}}{k_{B} T}\right) \approx 3.3
$$

where the factor $r_{\mathrm{oc} 2 / \text { ref }}$ takes into account the multiplicity of the amount of reference sites and relevant "oc2" sites.

Since for chemical trends only differences of solution energies are important, not the absolute values, the contribution of quantum mechanical zero point vibrations is neglected. In fact, they turn out to be only about $0.02 \mathrm{eV}$ as upper limit, for example, for $\mathrm{Fe}_{1-x} \mathrm{Mn}_{x}$ at $x=0,1$ as reported 
in [26]. Similar to the arguments in [26] we can therefore ignore this contribution.

In the calculations we have ignored magnetic effects. The antiferromagnetic double layer ordering has been shown to be the energetically most stable collinear configuration [34] and a good approximation to the true ground state in $\gamma \mathrm{Fe}$. However, we recall that two important effects of magnetism, namely, the repulsion of the hydrogen atoms by the magnetic moment and the increased interstitial volume due to the larger lattice constant with increasing magnetic orderingfrom nonmagnetic, via antiferromagnetic to ferromagneticare large by magnitude, but they mainly compensate each other. While this assumption is sufficient here, we refer to $[26,35]$ for details about the nonmagnetic approximation. For a recent review on ab initio modelling of magnetism in steels see [36]. Taking paramagnetism into account will be part of our future work to clarify the role of magnetic interactions on hydrogen bonding and diffusion.

3.1.2. Isotropic Deformation. In contrast to the calculations above, where we used a fixed pressure $P=0$, we now fix the volume of the supercell. This means that we evaluate the energies $E_{\mathrm{MH}}[\sigma]$ and $E_{\mathrm{M}}$ both at the same lattice constant. Specifically, for the equilibrium lattice constant of the system with hydrogen, the purely metallic system is therefore under tension and has a higher energy due to these elastic effects, compared to the results in Table 1, where both energies were calculated at their individual equilibrium volumes. Consequently, the solution energies are lower for the present fixed volume boundary conditions.

For isotropic deformations we strain the supercell equally in all three principal directions, which can be described by the diagonal transformation tensor

$$
\underline{\underline{\delta}}_{\text {hydro }}=(1+\delta) \cdot \underline{\underline{1}} .
$$

The resulting solution energies are shown in Figure 3 for the different configurations in Figures 1 and 2. The first observation is that to good accuracy all energies follow a linear behavior in the interval of investigated lattice constants $a_{\text {lat }}$ with almost the same slope, hence only having a parallel offset. This is in agreement with the generic picture that hydrogen mainly leads to an isotropic expansion of the stressfree system by an amount $\Delta V$, which differs only weakly for different metals [37]. Hence the coupling to an isotropic strain leads to an energy term proportional to $P \Delta V$. With the pressure $P$ being proportional to the deviation of the lattice constant from its equilibrium value, we therefore expect a linear dependence of the solution energy on the lattice constant.

We can further support this picture by a separation of the solution energy in a volumetric (elastic) and a chemical contribution, as indicated by the vertical and horizontal arrows in Figure 3, respectively. For the volumetric contribution we consider the hydrogen solution energy difference of the chemically identical system, but evaluated once at its equilibrium lattice constant and once at the lattice constant of the $\mathrm{Al}$ free reference system. Hence, this energy difference contains the release of mechanical work due to the

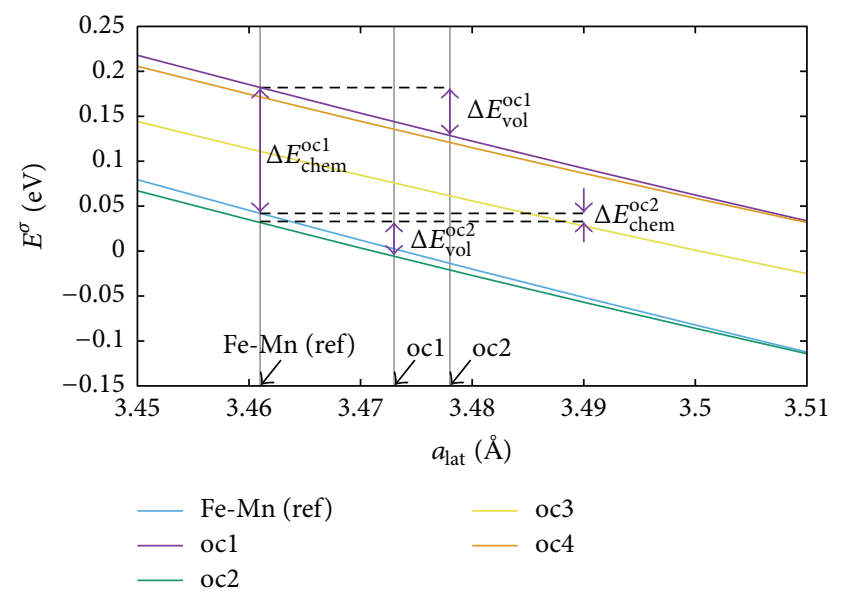

FIGURE 3: Hydrogen solution energies for the different octahedral interstitial sites in $\mathrm{Fe}_{24} \mathrm{Mn}_{8}$ and $\mathrm{Fe}_{24} \mathrm{Mn}_{8} \mathrm{Al}$ versus lattice constant $a_{\text {lat }}$. The different curves demonstrate the effect of aluminum on the solution energy. The latter is split into a chemical and a volumetric contribution; see main text. For all configurations the variation of the solution energy with the lattice constant is similar, as well as the volumetric contributions, whereas the chemical contributions differ significantly, indicating the strong preference of $\mathrm{H}$ to avoid an aluminum rich nearest neighbour environment. The vertical lines show the equilibrium lattice constants of the different configurations.

adjustment to the appropriate lattice constant. In contrast, the chemical contribution is calculated from the solution energy difference of two chemically different systems, namely, the $\mathrm{Fe}-\mathrm{Mn}-\mathrm{Al}$ and the Fe-Mn systems, but this time evaluated at the same lattice constant. Hence, this energy contribution expresses the energy difference for insertion of a single hydrogen atom into the same rigid atomic structure, but with different chemical environment due to the exchange of $\mathrm{Fe}$ by Al.

As can be seen from Figure 3 exemplarily for the configurations ocl and oc2, the volumetric contribution is basically identical, in agreement with the above argument, but the chemical contributions differ significantly. This difference is in line with the stress-free results in Table 1. Therefore, also under isotropic strain, we find that hydrogen prefers a direct environment which does not contain aluminum.

3.1.3. Tetragonal Deformation. Again, the starting point for the consideration is the system with a $\mathrm{Fe}_{24} \mathrm{Mn}_{8}$ supercell with cubic symmetry. By the substitution of one of the $\mathrm{Fe}$ atoms by $\mathrm{Al}$, the supercell experiences in equilibrium a shape relaxation with a slight tetragonal distortion. On a larger scale the $\mathrm{Al}$ atoms will be randomly distributed, and therefore the effects from the different specific arrangement will average out. As a result, the supercells will mainly preserve their cubic shape, which we take as basis for additional external tetragonal distortions.

In contrast to the isotropic deformations in the previous section we consider here a case where the distortion is volume-conserving, to clearly demonstrate the different 


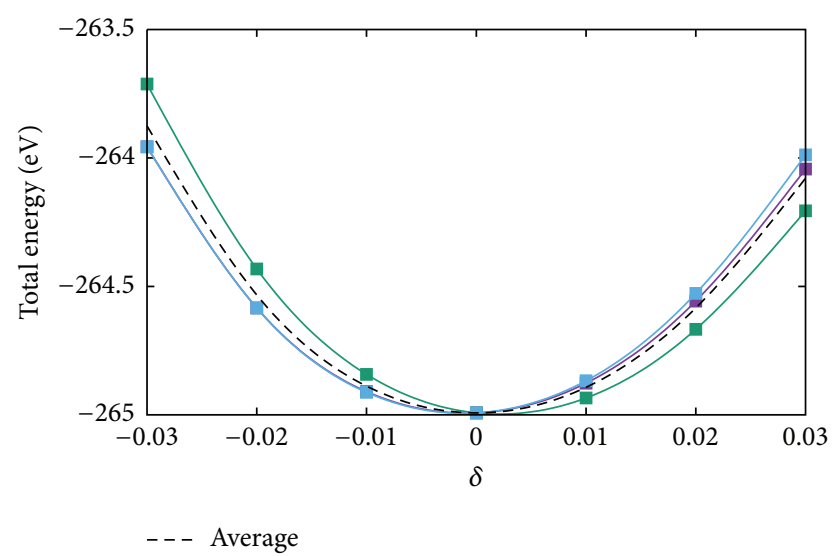

FIgURE 4: The total energy of the $\mathrm{Fe}_{23} \mathrm{Mn}_{8} \mathrm{Al}$ system with hydrogen at the ocl site. The coloured curves show the dependence of the energy on the orientation of the strain relative to the preferred elongation axis of the system. The dashed black curve shows the averaged energy.

behavior. For that, the supercell undergoes a transformation as described by the tensor

$$
\underline{\delta}_{\text {tetra }}=\left(\begin{array}{ccc}
1+\delta & 0 & 0 \\
0 & 1+\delta & 0 \\
0 & 0 & (1+\delta)^{-2}
\end{array}\right) \text {, }
$$

which is volume-conserving up to third order in the tetragonal strain $\delta$. The supercell is stretched in two principal directions and compressed in the other. The aforementioned dependence of the small intrinsic tetragonal distortion is equivalent to the application of different directions of the compression. The resulting energy curves as function of the tetragonal strain $\delta$ are shown in Figure 4 for the $\mathrm{Fe}_{23} \mathrm{Mn}_{8} \mathrm{AlH}$ supercell in configuration ocl. It shows the characteristic quadratic dependence of the elastic energy as function of strain when deformed from the cubic reference state. The dashed curve is the arithmetic means of the cases with the three different orientations of the principal axes and reflects the averaged elastic response for a larger system with random distributions. Such an averaging will therefore also be employed for the following calculations, while we still distinguish between the different configurations shown in Figure 2.

Similar to the isotropic case we computed the hydrogen solution energy as function of the tetragonal distortion, and the results are shown in Figure 5. Obviously, the behavior is very different than for the isotropic deformation in Figure 3. For that we note that the considered strain interval $\Delta \delta=0.06$ in the present plot is much larger than in the previous one, where we had $\Delta \delta \approx 0.017$. Still, the energetic variation is much smaller here, despite the larger strain interval. Together with the shape of the curves we can therefore conclude that strain effects to the solution energy are a higher order correction here. This is in agreement with the argument above that the interstitial hydrogen mainly leads to an isotropic

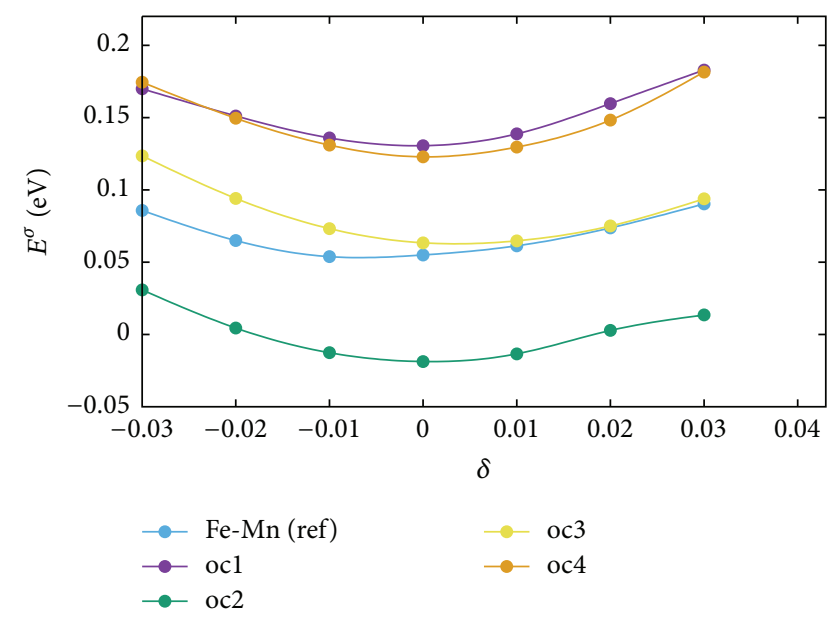

FIGURE 5: Hydrogen solution energy for the octahedral interstitial sites as function of the applied tetragonal distortion. The effect of the strain is much smaller than the chemical effect due to the substitution of Fe by Al.

expansion of the system, and therefore it only weakly interacts with a volume-conserving external strain.

As a result, the chemical influence of the $\mathrm{Al}$ atom is clearly dominant compared to the contributions from the tetragonal deformation. The hydrogen sites in the vicinity of the $\mathrm{Al}$ atom, ocl and oc4, exhibit solution energies which are not distinguishable within the accuracy limits of our approach. The oc3 site is comparably attractive as the reference site, while the oc2 site exhibits the shift of about $-60 \mathrm{meV}$ relative to the reference system basically over the full range of tetragonal deformations.

3.2. Hydrogen Diffusion. In this section we intend to obtain information on the hydrogen diffusion coefficient for different strain states and to understand the influence of aluminum. For that, we consider diffusion of hydrogen in the $\mathrm{Fe}-\mathrm{Mn}(-\mathrm{Al})$ matrix via trap site and Einstein diffusion. To discuss the influence of aluminum induced trapping we apply Oriani's theory [38], which is applicable when the trap sites and the regular lattice sites for the diffusing species are in local equilibrium. The term local equilibrium means that the kinetics of the equilibration of the regular sites and trap sites are described by sufficiently separated timescales. Then, important parameters are the solution energies and the trap densities. This allows drawing conclusions on the relative change of the diffusion coefficients depending on composition and strain.

For Einstein diffusion, we obtain estimates for the changes of the diffusion coefficients based on the diffusion barriers of the involved transition paths. We employ climbing image nudged elastic band calculations (CINEB) [39, 40], which search the saddle point of the reaction path, that is, the barrier between two connected sites.

In the spirit of the above discussion we use initial octahedral and final tetrahedral states of the reaction path with cubic cell symmetry and averaged lattice constants and therefore can refrain from using solid state nudged elastic 
TABLE 2: Energies at equilibrium volume, bulk modulus, and its derivative with respect to pressure as obtained from Murnaghan fits of the $a b$ initio data.

\begin{tabular}{lccc}
\hline System & $E_{0}(\mathrm{eV})$ & $B_{0}(\mathrm{GPa})$ & $\partial B / \partial P$ \\
\hline $\mathrm{Fe}_{24} \mathrm{Mn}_{8}$ & -265.581 & 176.6 & 4.78 \\
$\mathrm{Fe}_{24} \mathrm{Mn}_{8} \mathrm{H}$ & -268.922 & 175.6 & 4.70 \\
$\mathrm{Fe}_{23} \mathrm{Mn}_{8} \mathrm{Al}$ & -261.717 & 168.5 & 4.847 \\
$\mathrm{Fe}_{23} \mathrm{Mn}_{8} \mathrm{AlH}$ (ocl) & -264.992 & 168.3 & 4.763 \\
$\mathrm{Fe}_{23} \mathrm{Mn}_{8} \mathrm{AlH}$ (oc2) & -265.141 & 167.4 & 4.910 \\
\hline
\end{tabular}

band calculations [41]. The energy difference due to the averaged lattice constant-for example, $a_{\text {lat }}=3.502 \AA$ for $\mathrm{Mn}_{32} \mathrm{H}$ with hydrogen in an octahedral position and $a_{\mathrm{lat}}=$ $3.506 \AA$ for occupation of a tetrahedral site, leading to an averaged value $\bar{a}_{\text {lat }}=3.504 \AA-$ is in the range of $5 \mathrm{meV}$. We used $\mathrm{Mn}_{32} \mathrm{H}$ to test the validity of this approximation against available data on transition points and found good agreement [26]. Here $\bar{a}_{\text {lat }}$ for the $\mathrm{Fe}_{23} \mathrm{Mn}_{8} \mathrm{Al}$ system is chosen to minimize the error in the energies. For TWIP steels, it would need to be adjusted to other values. We also have checked the convergence of the CINEB calculations with respect to the number of images which initially discretize the transition path.

3.2.1. Stress-Free Systems. The oc2 sites are traps with a strain dependent binding energy. In absence of any deformation they have a depth of $60 \mathrm{meV}$, relative to the sites in the reference system $\mathrm{Fe}_{24} \mathrm{Mn}_{8}$. There are 20 possible hydrogen oc2 sites which are preferential and we assume that at most half of them are accessible due to the indirect hydrogen repulsion when placed in adjacent sites. This leads to the estimate of the trap density as $N_{t}=1.34 \times 10^{28} \mathrm{~m}^{-3}$, and the $N_{l}$ is the density of regular sites, with the ratio $N_{t} / N_{l} \approx 0.28$.

The apparent diffusivity relative to the trap-free diffusivity (aluminum-free) diffusivity is according to Oriani's theory

$$
\frac{D_{\mathrm{Fe}_{23} \mathrm{Mn}_{8} \mathrm{Al}}}{D_{\mathrm{Fe}_{24} \mathrm{Mn}_{8}}}=\frac{1}{1+\left(N_{t} / N_{l}\right) \exp \left(\left(E^{\mathrm{oc} 2}-E^{\mathrm{ref}}\right) / k_{B} T\right)},
$$

which is plotted in Figure 6 as function of temperature. We see that the presence of aluminum suppresses the hydrogen diffusivity in the room temperature range by about one order of magnitude.

3.2.2. Isotropic Deformation. Before discussing the influence of an isotropic deformation on trap site diffusion, we mention that differences in the bulk moduli between the hydrogenfree and hydrogen-loaded systems are typically small; see Table 2. Thus, equal strains correspond in good approximation to equal pressures.

Figure 6 shows in addition to the stress-free case the ratio of the trap site diffusion coefficient according to the Oriani model for isotropic compression $(\delta=-0.01)$ and tension $(\delta=0.01)$. This leads to a change of the diffusion coefficient relative to the case without aluminum by up to one order of magnitude at room temperature, based on the consideration of the oc2 traps. In general, a tensile strain increases the

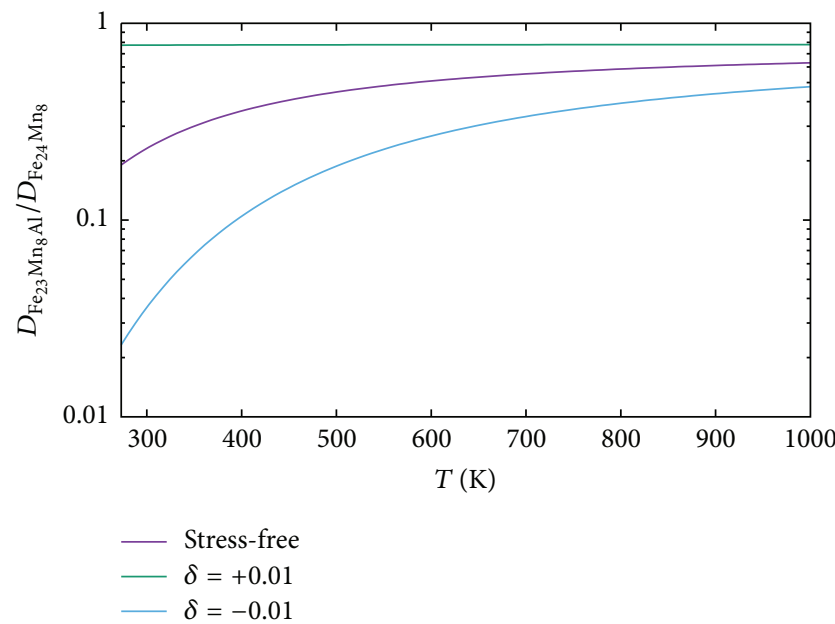

FIGURE 6: Ratio of the diffusion coefficients according to Oriani's theory as described by (6). The isotropic deformation leads to changes of the apparent diffusion coefficient by about one order of magnitude for a strain $\delta= \pm 0.01$ at room temperature. In all cases the presence of $\mathrm{Al}$ reduces the diffusion coefficient also by one order of magnitude in comparison to the $\mathrm{Fe}_{24} \mathrm{Mn}_{8}$ at ambient temperature.

diffusion coefficient, as the larger interatomic spacing allows for easier jumps of the interstitial hydrogen via a reduction of the energy barriers.

For Einstein diffusion we focus on the configurations ocl and oc 2 and the reference case of the supercell without $\mathrm{Al}$, as these are most illustrative in discussing the influence of the $\mathrm{Al}$ alloying with respect to deformation effects.

For isotropically strained systems with only one $\mathrm{Al}$ atom in the 32 atom supercell, the diffusion coefficient behaves as

$$
D \sim n_{\mathrm{tb}} a^{2} \exp \left(-\frac{E_{\text {Diff }}}{k_{B} T}\right),
$$

and both changes of the exponent and the preexponential factor for variations of composition and strain state have to be considered. Here, $n_{\mathrm{tb}}$ is the number of transition bonds to the next sites, $a$ is the distance to these sites, and $E_{\text {Diff }}$ is an effective diffusion barrier which reflects the dominant transitions contributing to the diffusion process.

In Figure 7 we show the barriers, that is, the energy difference $E_{\text {Diff }}$ between the transition and starting point, as function of lattice constant. The starting point is a hydrogen atom in position ocl, which then crosses a tetrahedral site close to the $\mathrm{Al}$ atom, with one $\mathrm{Fe}$ atom, two $\mathrm{Mn}$ atoms, and one $\mathrm{Al}$ atom as next neighbours. This initial site is relevant when local accumulations of Al yield a low density of available more attractive sites. The barrier of this transition close to the $\mathrm{Al}$ atom is increased by about $126 \mathrm{meV}$, which means that this transition will play a negligible role when other transitions are accessible. Under expansion, the system yields a substantial decrease of the barrier height.

The density of high-barrier transitions in the system with an $\mathrm{Al}$ atomic concentration of $1 / 32$ is still low, and therefore one cannot assume that they efficiently reduce the number of transition paths from the considered site in the spirit 


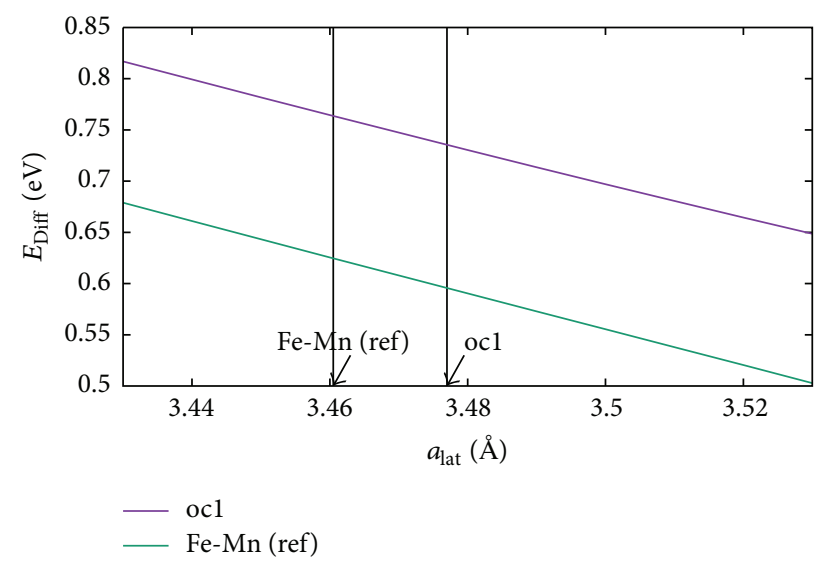

Figure 7: The diffusion barriers $E_{\text {Diff }}$ for the system before $\mathrm{Al}$ alloying, see the lower curve, and the site ocl in vicinity of the $\mathrm{Al}$ atom, see the upper curve. The grey bars mark from left to right the optimal volume of the reference site and ocl. We recall that, at zero pressure, the shift in barrier height due to substituting an Fe atom in the reference system by an $\mathrm{Al}$ atom is about $126 \mathrm{meV}$.

of shutting down percolation paths. Therefore, this leads only to a weak decrease of the preexponential factor $n_{\mathrm{tb}}$. However, when the number of high-barrier transition paths is increased, that is, when the atomic concentration of $\mathrm{Al}$ is increased to $2 / 32$ or $3 / 32$, the probability that only the high-barrier transitions along sites of type ocl are accessible from a considered site increases. Under these circumstances the energy barriers related to jump processes starting at ocl type positions become dominant, which leads to a change of the relevant exponential factor. It leads to a reduction of the diffusion coefficient relative to the aluminum-free system by a factor

$$
\exp \left(-\frac{E_{\text {Diff }}^{\text {ocl }}-E_{\text {Diff }}^{\text {ref }}}{k_{B} T}\right)
$$

which can reach $\sim 10^{-3}$ at room temperature. The contribution of direct jumps between octahedral sites can be expected to remain negligible even at appreciable strains. The energetic penalty of this transition of about $\approx 0.4-0.5 \mathrm{eV}$ relative to the octahedral-tetrahedral transition and the maximal effect of the strains of about $\approx 0.1-0.15 \mathrm{eV}$ suggest no change of the preferred diffusion path.

3.2.3. Tetragonal Strain. We can proceed in a similar way for systems under tetragonal strain. We distinguish between the three different possible strains relative to the elastically stiff axis, as they all appear in a random distribution of $\mathrm{Al}$ in an $\mathrm{Fe}-$ $\mathrm{Mn}-\mathrm{Al}$ alloy. As expected, the effect of the deformation on the trap site diffusion is weak; see Figure 8. At room temperature, the ratio of the apparent diffusion coefficients is changed by less than order of magnitude.

The picture is different when we consider the effect of the deformation on Einstein diffusion processes. As shown in Figure 9, variations in the relative orientation of the preferred elongation axis can result in a shift of the diffusion barriers by two orders of magnitude at low temperatures. The chemical

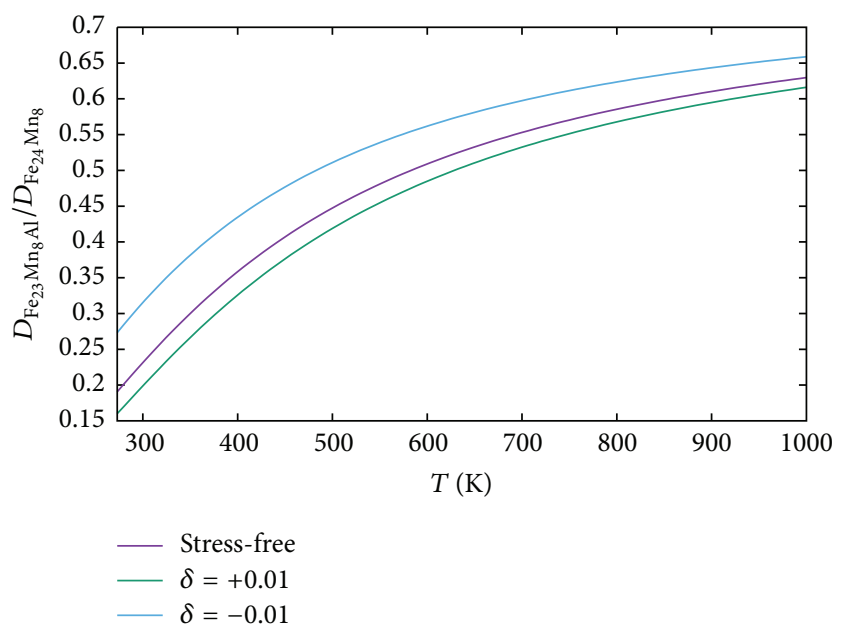

FIgURE 8: Ratio of diffusion coefficients corresponding to Oriani's theory for tetragonal strain. We note that the tetragonal deformation has a comparably weak effect on the apparent diffusivity. The ratio of the diffusion coefficients in the deformed systems varies by less than one order of magnitude.

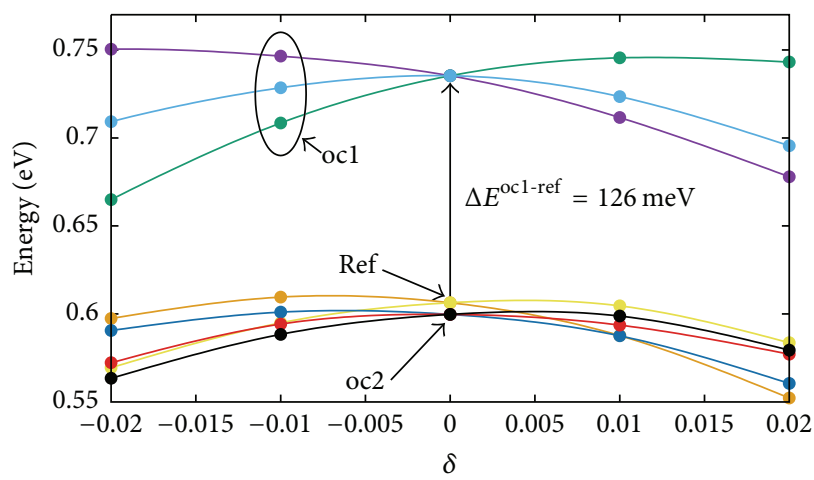

Figure 9: The diffusion barriers $E_{\text {Diff }}$ for the system before $\mathrm{Al}$ alloying, see the two middle curves in yellow and orange (ref), the diffusion barrier for the alloyed system from the octahedral site ocl in direct vicinity of an $\mathrm{Al}$ atom to a tetrahedral site with 1 neighbouring $\mathrm{Fe}, 2$ neighbouring $\mathrm{Mn}$, and 1 neighbouring $\mathrm{Al}$ atoms (three upper curves oc1), and the diffusion barriers for the alloyed system from the octahedral site oc2 distant to an $\mathrm{Al}$ atom to a tetrahedral site with 2 neighbouring Fe and 2 neighbouring $\mathrm{Mn}$ atoms (three lower curves oc2). Note that the shift in the diffusion barrier for the sites in the vicinity of the $\mathrm{Al}$ atom is about $126 \mathrm{meV}$. We recognise that the orientation of the tetragonal strain relative to the stiff axis of the system has appreciable influence especially for sites in the neighbourhood of the $\mathrm{Al}$ atom.

shift at zero pressure is larger, yielding a possible decrease of the diffusion coefficient in the vicinity of $\mathrm{Al}$ atoms up to three orders of magnitude. Again, the chemical contribution to the solution energy is dominant in the nonstressed state.

\section{Summary and Conclusions}

In the present paper we have discussed the influence of $\mathrm{Al}$ alloying and deformations on hydrogen absorption and 
diffusion in high manganese steels, represented by $\mathrm{Fe}_{24} \mathrm{Mn}_{8}$ and $\mathrm{Fe}_{23} \mathrm{Mn}_{8} \mathrm{Al}$ systems. In the considered configurations, the substitution with Al leads to two counteracting effects on the hydrogen dissolved on interstitial sites in the matrix. On the one hand, at low $\mathrm{Al}$ concentrations of about 3 at\%, the number of trap sites, which increase the hydrogen solubility and decrease the diffusivity, is large. On the other hand, at larger $\mathrm{Al}$ concentrations an effective blocking of paths for the hydrogen diffusion could lead to a drastic reduction of the diffusivity, caused by the high barrier of the transition path between octahedral and tetrahedral sites in the direct vicinity of aluminum atoms. Here we note that the high Mn contents in TWIP steels allow for high $\mathrm{Al}$ additions without destabilizing the austenite.

In general, the influence of volumetric changes during isotropic deformations has larger influence on the diffusivity of hydrogen compared to volume preserving tetragonal strains. While the volumetric deformation typically results in a linear dependence of the solution energy on the volume, the influence of tetragonal strains yields effects of second order. This is in agreement with the notion that hydrogen leads to an isotropic expansion of the host alloy.

Spatially inhomogeneous Al distributions lead to an orientation dependent influence of a tetragonal distortion on the diffusion of hydrogen. High local aluminum concentrations have the potential to reduce the diffusion coefficient locally by up to three orders of magnitude in the room temperature regime. For a careful investigation of this effect, large scale Monte Carlo simulations with an emphasis on percolation effects may shed further light on this issue.

\section{Competing Interests}

The authors declare that they have no competing interests.

\section{Acknowledgments}

This work has been supported by the Collaborative Research Center (SFB) 761 Steel $a b$ initio of the German Research Foundation (DFG). The authors gratefully acknowledge the computing time granted on the supercomputer JURECA at the Jülich Supercomputing Centre (JSC). Discussions with Tilmann Hickel are highly appreciated by the authors.

\section{References}

[1] M. Koyama, H. Springer, S. V. Merzlikin, K. Tsuzaki, E. Akiyama, and D. Raabe, "Hydrogen embrittlement associated with strain localization in a precipitation-hardened $\mathrm{Fe}-\mathrm{Mn}$ Al-C light weight austenitic steel," International Journal of Hydrogen Energy, vol. 39, no. 9, pp. 4634-4646, 2014.

[2] C. Scott, S. Allain, M. Faral, and N. Guelton, "The development of a new Fe-Mn-C austenitic steel for automotive applications," Revue de Metallurgie, vol. 103, no. 6, pp. 293-302, 2006.

[3] B. De Cooman, J. Kim, and K.-G. Chin, High Mn TWIP Steels for Automotive Applications, InTech, Rijeka, Croatia, 2011.

[4] D. Barbier, N. Gey, S. Allain, N. Bozzolo, and M. Humbert, "Analysis of the tensile behavior of a TWIP steel based on the texture and microstructure evolutions," Materials Science and Engineering: A, vol. 500, no. 1-2, pp. 196-206, 2009.

[5] M. Koyama, T. Sawaguchi, and K. Tsuzaki, "TWIP effect and plastic instability condition in an Fe-Mn-C austenitic steel," ISIJ International, vol. 53, no. 2, pp. 323-329, 2013.

[6] I. Gutierrez-Urrutia and D. Raabe, "Dislocation and twin substructure evolution during strain hardening of an Fe-22 wt.\% Mn-0.6 wt.\% C TWIP steel observed by electron channeling contrast imaging," Acta Materialia, vol. 59, no. 16, pp. 64496462, 2011.

[7] D. R. Steinmetz, T. Jäpel, B. Wietbrock et al., "Revealing the strain-hardening behavior of twinning-induced plasticity steels: theory, simulations, experiments," Acta Materialia, vol. 61, no. 2, pp. 494-510, 2013.

[8] M. Koyama, T. Sawaguchi, T. Lee, C. S. Lee, and K. Tsuzaki, "Work hardening associated with $\varepsilon$-martensitic transformation, deformation twinning and dynamic strain aging in $\mathrm{Fe}-17 \mathrm{Mn}-$ $0.6 \mathrm{C}$ and Fe-17Mn-0.8C TWIP steels," Materials Science and Engineering: A, vol. 528, no. 24, pp. 7310-7316, 2011.

[9] S. Allain, J.-P. Chateau, and O. Bouaziz, "A physical model of the twinning-induced plasticity effect in a high manganese austenitic steel," Materials Science and Engineering: A, vol. 387389, no. 1-2, pp. 143-147, 2004.

[10] G. Frommeyer, U. Brüx, and P. Neumann, "Supra-ductile and high-strength manganese-TRIP/TWIP steels for high energy absorption purposes," ISIJ International, vol. 43, no. 3, pp. 438446, 2003.

[11] A. Grajcar, S. Kołodziej, and W. Krukiewicz, "Corrosion resistance of high-manganese austenitic steels," Archives of Materials Science and Engineering, vol. 41, no. 2, pp. 77-84, 2010.

[12] R. Frohmberg, W. Barnett, and A. Troiano, "Delayed failure and hydrogen embrittlement in steel," Defense Technical Information Center (DTIC Document) 54-320, 1954.

[13] T. P. Perng and C. J. Altstetter, "Comparison of hydrogen gas embrittlement of austenitic and ferritic stainless steels," Metallurgical Transactions A, vol. 18, no. 1, pp. 123-134, 1987.

[14] I.-J. Park, K.-H. Jeong, J.-G. Jung, C. S. Lee, and Y.-K. Lee, "The mechanism of enhanced resistance to the hydrogen delayed fracture in $\mathrm{Al}$-added $\mathrm{Fe}-18 \mathrm{Mn}-0.6 \mathrm{C}$ twinning-induced plasticity steels," International Journal of Hydrogen Energy, vol. 37, no. 12, pp. 9925-9932, 2012.

[15] M. Koyama, E. Akiyama, and K. Tsuzaki, "Effects of static and dynamic strain aging on hydrogen embrittlement in TWIP steels containing al," ISIJ International, vol. 53, no. 7, pp. 12681274, 2013.

[16] M. Koyama, E. Akiyama, T. Sawaguchi, D. Raabe, and K. Tsuzaki, "Hydrogen-induced cracking at grain and twin boundaries in an Fe-Mn-C austenitic steel," Scripta Materialia, vol. 66, no. 7, pp. 459-462, 2012.

[17] M. Koyama, E. Akiyama, K. Tsuzaki, and D. Raabe, "Hydrogenassisted failure in a twinning-induced plasticity steel studied under in situ hydrogen charging by electron channeling contrast imaging," Acta Materialia, vol. 61, no. 12, pp. 4607-4618, 2013.

[18] M. Koyama, E. Akiyama, and K. Tsuzaki, "Hydrogen embrittlement in a Fe-Mn-C ternary twinning-induced plasticity steel," Corrosion Science, vol. 54, no. 1, pp. 1-4, 2012.

[19] A. Grajcar, "Corrosion resistance of high-Mn austenitic steels for the automotive industry," Corrosion Resistance, pp. 353-376, 2012. 
[20] A. Grajcar, R. Kuziak, and W. Zalecki, "Third generation of AHSS with increased fraction of retained austenite for the automotive industry," Archives of Civil and Mechanical Engineering, vol. 12, no. 3, pp. 334-341, 2012.

[21] H. Hata, T. Murakami, A. Ibano, F. Yuse, and J. Kinugasa, "Coldrolled steel sheet," US Patent 8,876,986, 2014.

[22] T. Michler and J. Naumann, "Hydrogen environment embrittlement of austenitic stainless steels at low temperatures," International Journal of Hydrogen Energy, vol. 33, no. 8, pp. 21112122, 2008.

[23] M. Louthan Jr., R. McNitt, and R. Sisson Jr., "Environmental degradation of engineering materials in hydrogen," Tech. Rep., Laboratory for the Study of Environmental Degradation of Engineering Materials, Virginia Polytechnic Institute and State University, Blacksburg, Va, USA, 1981.

[24] C. San Marchi, B. P. Somerday, X. Tang, and G. H. Schiroky, "Effects of alloy composition and strain hardening on tensile fracture of hydrogen-precharged type 316 stainless steels," International Journal of Hydrogen Energy, vol. 33, no. 2, pp. 889-904, 2008.

[25] V. G. Gavriljuk, V. N. Shivanyuk, and J. Foct, "Diagnostic experimental results on the hydrogen embrittlement of austenitic steels," Acta Materialia, vol. 51, no. 5, pp. 1293-1305, 2003.

[26] L. Ismer, T. Hickel, and J. Neugebauer, " $A b$ initio study of the solubility and kinetics of hydrogen in austenitic high Mn steels," Physical Review B, vol. 81, no. 9, Article ID 094111, 2010.

[27] J. von Appen, R. Dronskowski, A. Chakrabarty, T. Hickel, R. Spatschek, and J. Neugebauer, "Impact of $\mathrm{Mn}$ on the solution enthalpy of hydrogen in austenitic Fe-Mn alloys: a firstprinciples study," Journal of Computational Chemistry, vol. 35, no. 31, pp. 2239-2244, 2014.

[28] G. Kresse and J. Furthmüller, "Efficient iterative schemes for ab initio total-energy calculations using a plane-wave basis set," Physical Review B, vol. 54, no. 16, pp. 11169-11186, 1996.

[29] P. E. Blöchl, "Projector augmented-wave method," Physical Review B, vol. 50, no. 24, pp. 17953-17979, 1994.

[30] J. P. Perdew, K. Burke, and M. Ernzerhof, "Generalized gradient approximation made simple," Physical Review Letters, vol. 77, no. 18, pp. 3865-3868, 1996.

[31] H. J. Monkhorst and J. D. Pack, "Special points for Brillouinzone integrations," Physical Review B, vol. 13, no. 12, pp. 51885192, 1976.

[32] M. Methfessel and A. T. Paxton, "High-precision sampling for Brillouin-zone integration in metals," Physical Review B, vol. 40, no. 6, pp. 3616-3621, 1989.

[33] A. Sieverts, "Absorption of gases by metals," Zeitschrift für Metallkunde, vol. 21, pp. 37-46, 1929.

[34] E. Sjöstedt and L. Nordström, "Noncollinear full-potential studies of $\gamma$-Fe," Physical Review B, vol. 66, no. 1, Article ID 014447, 10 pages, 2002.

[35] E. J. Song, H. K. D. H. Bhadeshia, and D.-W. Suh, "Interaction of aluminium with hydrogen in twinning-induced plasticity steel," Scripta Materialia, vol. 87, pp. 9-12, 2014.

[36] F. Körmann, T. Hickel, and J. Neugebauer, "Inuence of magnetic excitations on the phase stability of metals and steels," Current Opinion in Solid State and Materials Science, vol. 20, no. 2, pp. 77-84, 2016.

[37] Y. Fukai, The Metal-Hydrogen System, Springer, Berlin, Germany, 2nd edition, 2005.

[38] R. A. Oriani, "The diffusion and trapping of hydrogen in steel," Acta Metallurgica, vol. 18, no. 1, pp. 147-157, 1970.
[39] G. Henkelman, B. P. Uberuaga, and H. Jónsson, "Climbing image nudged elastic band method for finding saddle points and minimum energy paths," The Journal of Chemical Physics, vol. 113, no. 22, pp. 9901-9904, 2000.

[40] G. Henkelman and H. Jónsson, "Improved tangent estimate in the nudged elastic band method for finding minimum energy paths and saddle points," The Journal of Chemical Physics, vol. 113, no. 22, pp. 9978-9985, 2000.

[41] D. Sheppard, P. Xiao, W. Chemelewski, D. D. Johnson, and G. Henkelman, "A generalized solid-state nudged elastic band method," The Journal of Chemical Physics, vol. 136, no. 7, Article ID 074103, 2012. 

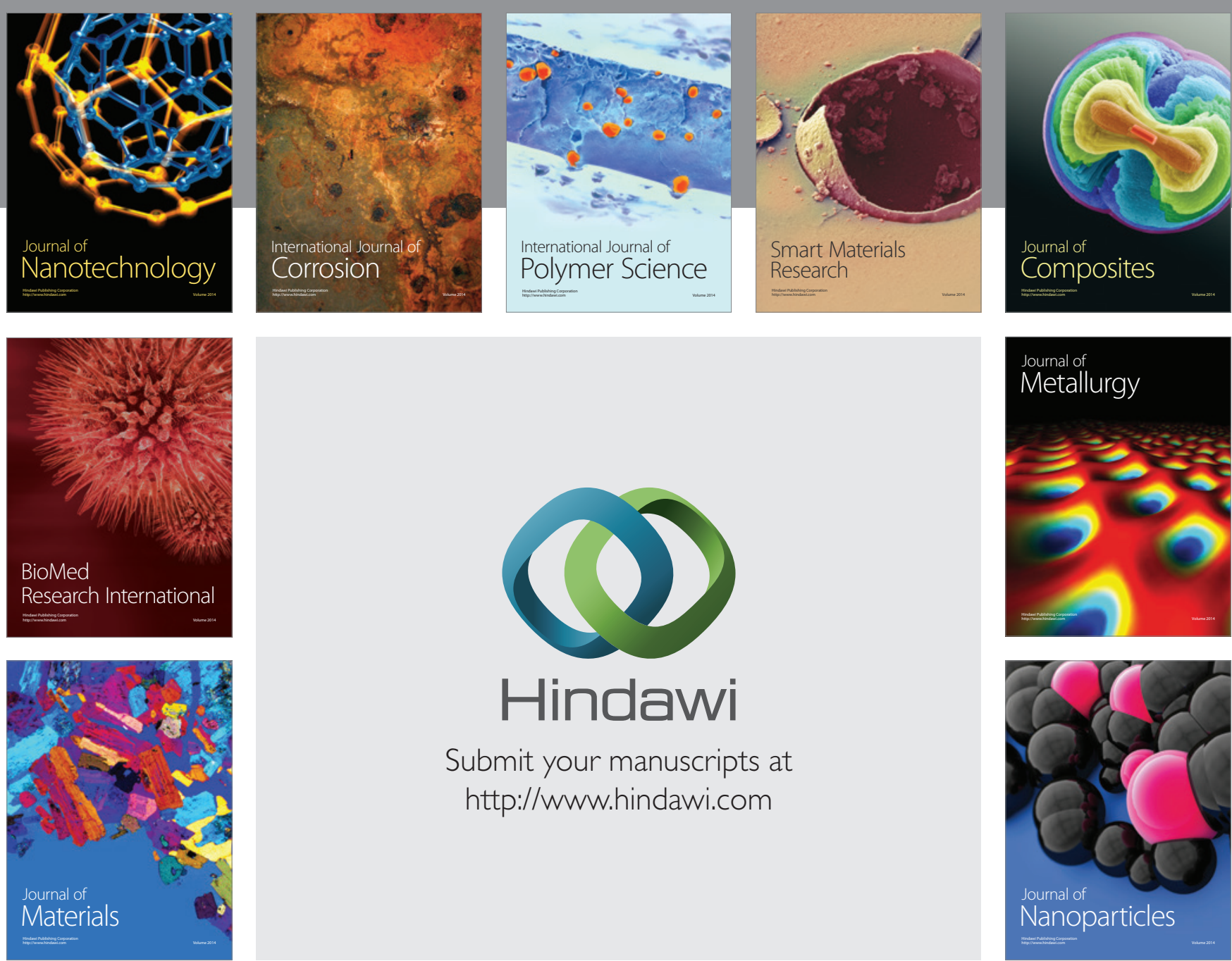

\section{Hindawi}

Submit your manuscripts at

http://www.hindawi.com

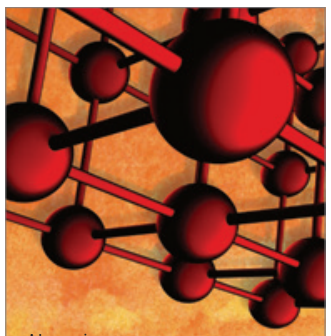

Materials Science and Engineering
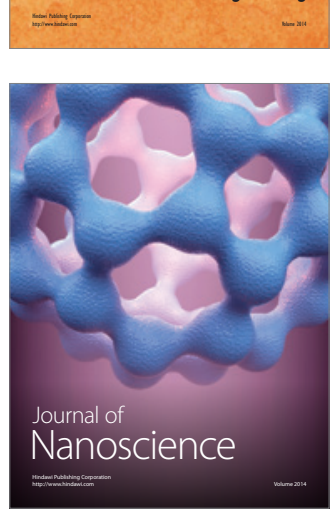
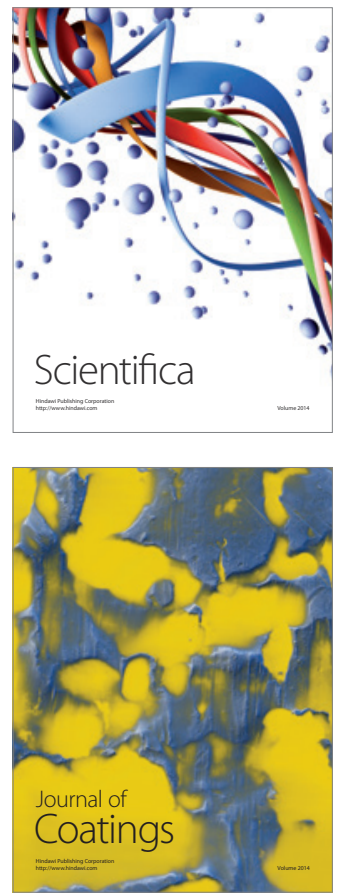
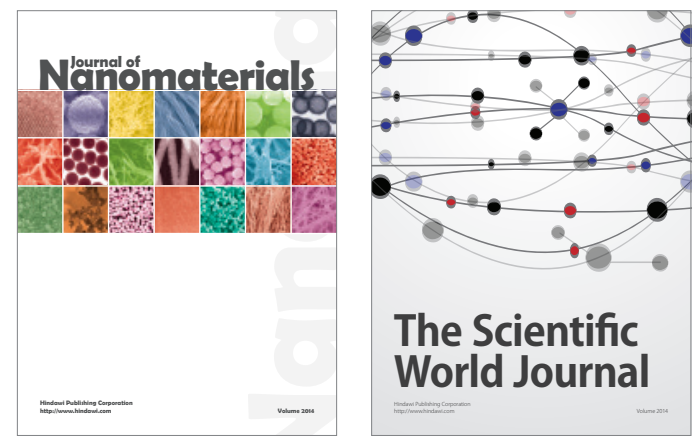

The Scientific World Journal
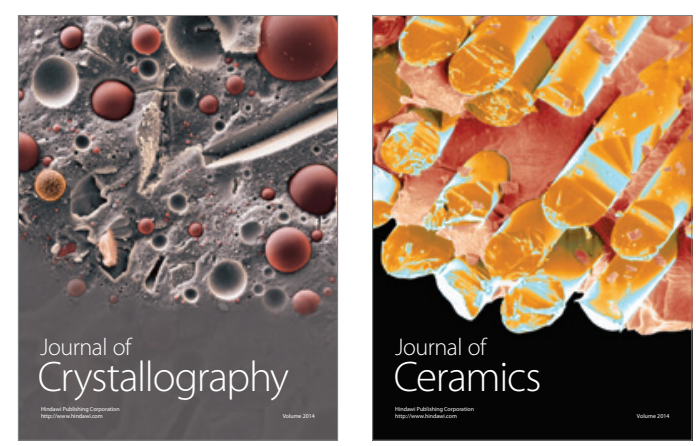
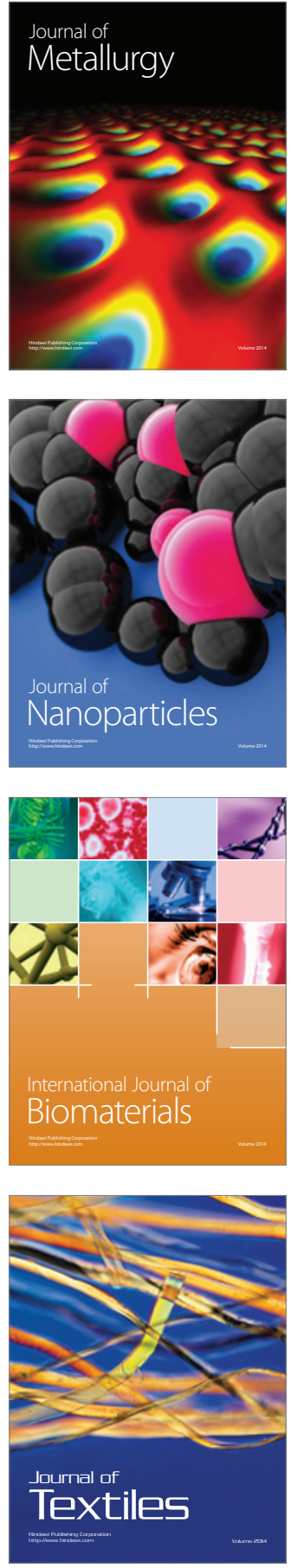